\title{
Collaborative Authoring of Adaptive Learning Resources: Opportunities and Challenges
}

\author{
Dade Nurjanah, Hugh C. Davis, and Thanassis Tiropanis \\ The University of Southampton \\ Southampton, UK \\ $\{$ dn08r, hcd, tt2 $\} @$ ecs.soton.ac.uk
}

\begin{abstract}
One issue regarding adaptive learning systems is the provision of learning resources that consist of domain knowledge, pedagogical knowledge with adaptation rules, and learning content. Challenges emerge concerning the lack of usability, interoperability, efficiency and collaboration. This paper critically discusses an enhanced collaborative authoring approach to developing adaptive learning resources. The main motivation behind the proposed approach is the evidence that creating adaptive learning resources is a complex, timeconsuming process. Therefore, teachers must work together to reduce individual efforts. Until recently, collaborative work has not been strongly supported by existing authoring tools for adaptive learning. The contribution of this paper is twofold: the analysis of current authoring tools for learning and the proposed approach, which addresses the importance of collaboration and reuse in order to improve authors' awareness of collaborative work.
\end{abstract}

Keywords- CSCW (Computer-Supported Collaborative Work); adaptive learning; IMS Learning Design; awareness; History; Note.

\section{INTRODUCTION: WHY COLLABORATION?}

Recent developments in the field of learning systems have led to enhanced learning systems, which consider learners' characteristics when making pedagogical decisions. This improvement produces adaptation in various ways that offer students a range of appropriate learning options. In adaptive learning, in which learners' characteristics determine which topics are presented and how they are delivered, learning resources also consist of a learner model and an adaptation model. Adaptive learning, however, gives teachers the responsibility of providing learning resources that are complex and large, with various elements of domain knowledge, pedagogical knowledge, learner information, learning content and adaptation rules. This is because developing adaptive learning resources is a complex and time-consuming process. As a consequence, teachers must prepare a large volume of learning resources for online as well as offline learning activities. The complexity of the endeavours needed to develop learning resources has been studied in former research [1].

Because of the sheer size and complexity of the learning space required for adaptive learning, it is difficult for just one or two people to develop such a space. Teachers need to work together in order to reduce individual effort. Kearsley [2] argues that, although teachers can work individually on preparing courses, they will need a great deal of time to understand all the important aspects of learning, to develop instructional design and to create learning materials. However, a challenge arises in terms of providing authoring toolsthat support collaboration. Our preliminary work on a comparison of current authoring tools for learning shows that collaboration is not supported. They support reuse, but that is not an appropriate approach to group work. Group work is not merely a collection of people individually working to perform a task. Each author should understand what other authors do, why a learning object should be created and how the authoring process is proceeding.

Research studies have shown that instructional designers carry out brainstorming and discussions with their colleagues in designing instructional strategies [3-5]. These studies also showed that such interactions have more influence on their work than theories on instructional design. Some current authoring tools enable authors to reuse objects and to communicate with others using instant messaging or email. However, since such communication tools or features are not integrated with objects, effort is required to identify which thread a particular topic is being discussed on.

This paper discusses a work-in-progress that could one day be used for collaborative authoring of adaptive learning resources. The main contribution of this paper is twofold: First, it provides a comparison of existing authoring tools for learning resources, including MOODLE, AHA!, MOT, GAT and ReCourse. Second, it presents a collaborative authoring approach for adaptive learning resources. The main questions addressed in this paper in terms of authoring adaptive learning resources are: a) What are the problems that appear in the authoring of adaptive learning resources? b) What are the advantages and drawbacks of existing authoring tools? c) What collaborative methods are needed for authoring adaptive learning resources?

The rest of this paper is organized as follows. Section 2 discusses related work from former research studies on communication and coordination features in CSCW and learning standards. Section 3 presents a comparison of existing authoring tools for learning system resources. Section 4 describes the proposed collaborative authoring approach. Finally, Section 5 presents conclusions and future work.

\section{RELATED WORK}

Collaborative work has been successfully implemented in other areas. There is evidence in studies on CSCW that can be 
adopted in order to improve authoring tools for adaptive learning. First, imposing appropriate coordination techniques in collaborative work improves the efficiency of the work. Former studies have divided coordination into two categories: implicit coordination and explicit coordination [6, 7]. Second, some CSCW features have successfully improved authors' awareness [8, 9]. In Wikipedia, the features are implemented in Talkpages in which authors can hold discussions and Logs that record what authors have done to which documents.

\section{A. Awareness in CSCW}

Former research has reported the implementation of CSCW for various objects, such as documents [10], knowledge [11], papers [8] and learning resources $[12,13]$. Other studies have focused on enhancing the collaborative authoring process and ouput by enhancing authors' awareness and implementing communication features for coordination, commenting or discussion among authors in synchronous as well as asynchronous collaborative authoring. The implementation of such features is varied, involving face-to-face meetings [14, 15], direct messaging [15], talk pages [6, 7] and annotation $[11,16]$. In addition to such features, some studies have investigated the importance of recording provenance information in order to enhance authors' awareness of the dynamics of the authoring process $[11,17]$.

It has been proven in former studies that increasing authors' awareness can improve the quality of learning resources. Learning resources produced by collaborative work will be of higher quality than those created by individual work. Past research studies indicated the need for collaboration through social annotation [18]. In addition, collaboration can increase the possibility of learning resources being continuously updated, thus ensuring that they remain relevant to students' needs [19]. The quality of learning resources, however, is not merely influenced by how many authors contribute, but by how the collaboration is carried out. Gaining consensus [20], which includes all authors' insights and visions, is a way forward in collaboration that will produce meaningful results. The requirements for collaborative work have been presented in a former paper [21].

There is a correlation between authors' awareness and coordination. Coordination becomes easier as authors' awareness increases, although awareness is not the only catalyst for improved coordination[15]. Former studies have demonstrated that coordination will work effectively when the coordination method used is appropriate to the number of authors and the independency of tasks $[6,7,14]$. These studies concluded that explicit coordination in which authors discuss and brainstorm to reach some consensus on an authoring plan is suitable for large workgroups with highly independent tasks. On the other hand, authors do not discuss implicit coordination in advance. It is implemented in the authoring process structuring built by one or a few authors. Implicit planning is to be used in small workgroups with highly dependent tasks.

\section{B. Learning Standards for Collaborative Work}

Reusability has been suggested as one quality that learning resources should have [22]. It is related to the flexibility of resources, their interoperability for repurposing resources, and their suitability to other tools, such as other authoring tools, Learning Management Systems (LMSs) or players. A way to achieve reusability is by representing learning resources using a learning standard that provides mechanisms and formats for describing, packaging, sequencing and delivering content or activities. Until recently, although there have been a number of learning standards, only a few supported adaptation, such as Simple Sequencing (SS) and Learning Design (LD). Contrary to SS, in which adaptation receives only limited supported, LD moderately supports adaptation, including flow-based (navigation), content-based and interactive problem solving support-based adaptations [23]. With LD, teachers are able to create their own domain model; pedagogical matters, including adaptation rules; and learner model, which combines learning parameters and the learner's achievements in a way that can be used in adaptation rules.

\section{A PRELIMINARY WORK: A COMPARISON OF EXISTING AUTHORING TOOLS}

This section describes the advantages and disadvantages of current authoring tools for learning resources, including AHA!, MOT, GAT, MOODLE and ReCourse. The comparison of such tools considers aspects including adaptation support, the use of learning standards, collaboration support and communication features.

\section{A. AHA! Editor}

AHA! Editor is a part of AHA! that implements the general structure of Adaptive Educational Hypermedia (AEH) systems, which maintains concepts, concept relationships and adaptation rules [24]. It supports content-based adaptation, including adaptive navigation and adaptive presentation. Adaptation rules in AHA! are embeded in learning material files as assertions that will be executed along with the learner model to perform adaptive content and adaptive link presentations.

AHA! enables authors to reuse existing materials. However, it does not support collaboration or communication among authors. Another disadvantage of AHA! is that the output is tightly bound to its inference engine, thus making it difficult deliver to other AEH systems.

\section{B. My Online Teacher (MOT)}

MOT [25] is an general-purpose online authoring tool that produces reusable objects in an XML-based format. Like AHA!, there is no feature for collaboration and author communication in MOT, but it enables learning material reuse. Regarding adaptation support, MOT is better than AHA! because it supports content-based adaptation, like AHA!, and also learning-flow adaptation. MOT implements a five-layer authoring model, consisting of a Domain Model, Goal Model, User Model, Adaptation Model and Presentation Model. It does not apply any learning standard, because it defines its own knowledge representation, LAG (Layers of Adaptive Granularity).

\section{GRAPPLE Authoring Tool (GAT)}

GAT [26] categorizes authored resources into three types: Domain Model (DM), Concept Relational Types (CRT) and Concept Adaptation Model (CAM). It also supports learningflow-based adaptation or adaptive sequence. One advantage of 
GAT lies in CRT, which maintains abstract descriptions of pedagogical relationships. It represents classes of relationships, without specifying which concepts are involved. Therefore, it offers high reusability in that it enables authors to reuse existing CRT.

Creating learning content is not supported in GAT. However, it enables authors to link concepts in the Domain Model to learning content from various sources. A drawback of GAT is that CRT authoring is complicated for authors in that authors must define user model variables and some of the codes for adaptation. Novice users and those who have never worked in programming will need time to understand and create their own CRT. To conclude, GAT does not apply any learning standard, and it does not support collaborative work.

\section{MOODLE}

MOODLE (http://moodle.org/) is a course/learning management system (C/LMS) that provides authoring for various objects, such as syllabi, teaching plans and content packages. One advantage of MOODLE is that it provides many formats, such as SCORM or IMS content packaging. A further advantage lies in its capability to enable authors to reuse existing materials by providing an import function.

MOODLE supports collaborative work in the form of wikis, glossaries, and database modules that many authors can contribute to. Communication among authors is enabled through email/internal messaging, synchronous chat and asynchronous discussion. The disadvantage of such features is that they are not attached to learning objects. Teachers must search within the message/email repository or chat histories to find discussions related to a particular topic. Even with such advantages, however, MOODLE is not suitable for authoring adaptive learning resources. It does not provide any function that enables authors to include adaptation in learning materials.

\section{E. ReCourse}

ReCourse (http://tencompetence-project.bolton.ac.uk) is an authoring tool for authoring at IMS LD as well as providing functions for managing adaptation rules and constraints. The main advantage of ReCourse lies in its use of IMS LD to represent and manage its output. As ReCourse uses IMS LD, it supports three kinds of adaptation: learning flow-based adaptation, content-based adaptation and problem solving support adaptation.ReCourse hides the complex structure of learning design from authors during the authoring process. Authors interact with the objects of authoring through usable interfaces, with graphs to visualise what has been created in the authoring process. In addition, downloadable output enables teachers to enrich or repurpose it using other authoring tools. The drawbacks of ReCourse arise from its characteristics as a standalone application that prevents authors from working collaboratively. However, ReCourse enables authors to reuse existing content from other sources without giving any preference.

\section{A NEW APPROACH TO COLLABORATIVE AUTHORING FOR ADAPTIVE LEARNING RESOURCES}

Due to the drawbacks of current authoring tools for adaptation and the successfully implemented collaborative work in former research studies, a new approach to collaborative adaptation authoring is proposed. The new approach, Collaborative Adaptation Authoring (CAA), addresses the importance of including a collaborative feature in adaptation authoring in addition to the adaptive learning requirements. It is based on the consideration that human-tohuman interaction can improve adaptation authoring, as was proven in former research studies on the collaborative authoring of various objects.

\section{A. Main Concerns}

The CAA approach is designed for asynchronous, distributed collaboration that enables authors to work collaboratively towards a common goal from different places and at different times. It is designed for a small group of instructional designers or instructors/teachers to collaboratively create adaptive learning resources. In this approach, it is recommended that teachers do not spend their timecreating learning materials. Instead, it is suggested that they use materials from existing open content systems. Teachers must focus on creating learning activities, learning environments, learning flows, learner models and learning rules. All these objects are structured in an IMS manifest as the main output of the authoring process. Other outputs are Note, which contains all the discussions that occurred among the authors, and History, which records all updates made by the authors.

One critical issue in collaborative authoring is the awareness of the authors, which can take several forms [15]. This approach addresses personal awareness, informal awareness and group awareness.

\section{Personal awareness}

Personal awareness relates to authors' knowledge of what they have done in the collaborative authoring process. It will help authors to review their past work and plan future work. To support personal awareness, a feature named History, whichrecords all updates performed during the authoring process, is implemented. History saves information about how the authoring process is proceeding, what authors have done and which objects they have made or updated. This informationcan help novice users understand how the authoring process has progressed.

\section{Informal awareness}

Informal awareness is related to the provision of information about who is in the collaborative work environment and what other authors have done during particular periods of time. In addition to History, the CAA approach implements Note,which enables authors to communicate and add comments. This is attached to every authored object. Therefore, authors can easily find all annotations and comments that relate to a particular topic and know what other authors have done recently. For novice authors, such information can help them to understand the authoring process.

3. Group awareness

Group awareness relates to authors' awareness of other authors's roles and movements and the groupwork process. In addition to Note and History, the authoring plan can also increase group awareness of authors. As the CAA approach is designed for small groups, planning can be implemented with explicit coordination as well as an implicit method. 
Both will work efficiently. Authors can carry out explicit planning using Note attached to the whole learning design in order to discuss and create some consensus before outlining learning design.

\section{B. Authoring Scenario}

The approach focuses on the creation and provision of information to enhance authors' awareness. The collaborative features implemented are Note, which enables authors to comment, and History, which maintains a record of updates made during the authoring process. Although collaborative work is the main concern, the proposed approach also addresses the reusability of output. IMS LD is chosen torepresent learning resources, because that is the learning standard that most supports adaptive learning.

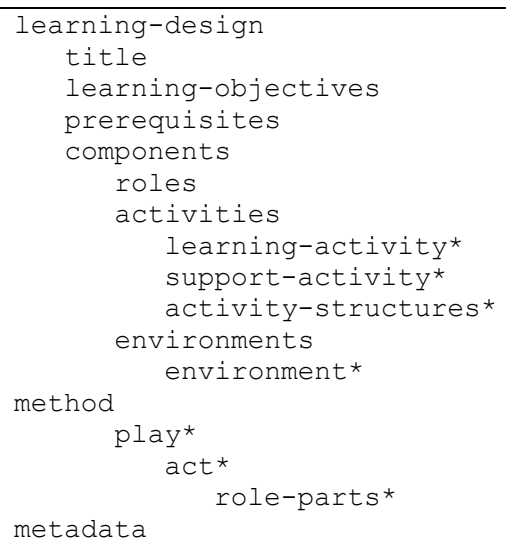

Figure 1. The IMS LD

One concern indeveloping a collaborative authoring approach is related to coordination. The CAA approach applied implicit coordination due to the IMS LD structure (Figure 1), which indicates a similarity to process structurescreated in former collaborative authoring research that applied implicit coordination. Therefore, in this case, implicit coordination is considered to be more suitable than explicit coordination. With implicit coordination, an author first initiates the authoring process by creating the top level of LD as a skeleton of the learning scenario, including a title, learning-objectives, prerequisites and probably set modules/phases. Such initial LD is aimed at guiding authors regarding what should and should not be created during the authoring. Note implemented and attached to the structure enables authors to attach comments and explanations. It can help authors understand more about the authoring process and output. Other authors can modify the initial LD or adding learning/support activities, resources and environments.

In order to evaluate the proposed authoring approach, a collaborative authoring tool for adaptive learning resources has been implemented. ReCourse was selected to be extended, rather than other authoring tools, considering that transforming a current authoring tool into a collaborative one is more appropriate and efficient than building a tool from scratch. As open source software, ReCourse is free to be extended. Another advantage of ReCourse is due to the languageit uses, IMS LD, which supports interoperability, reusability, adaptation and completeness.

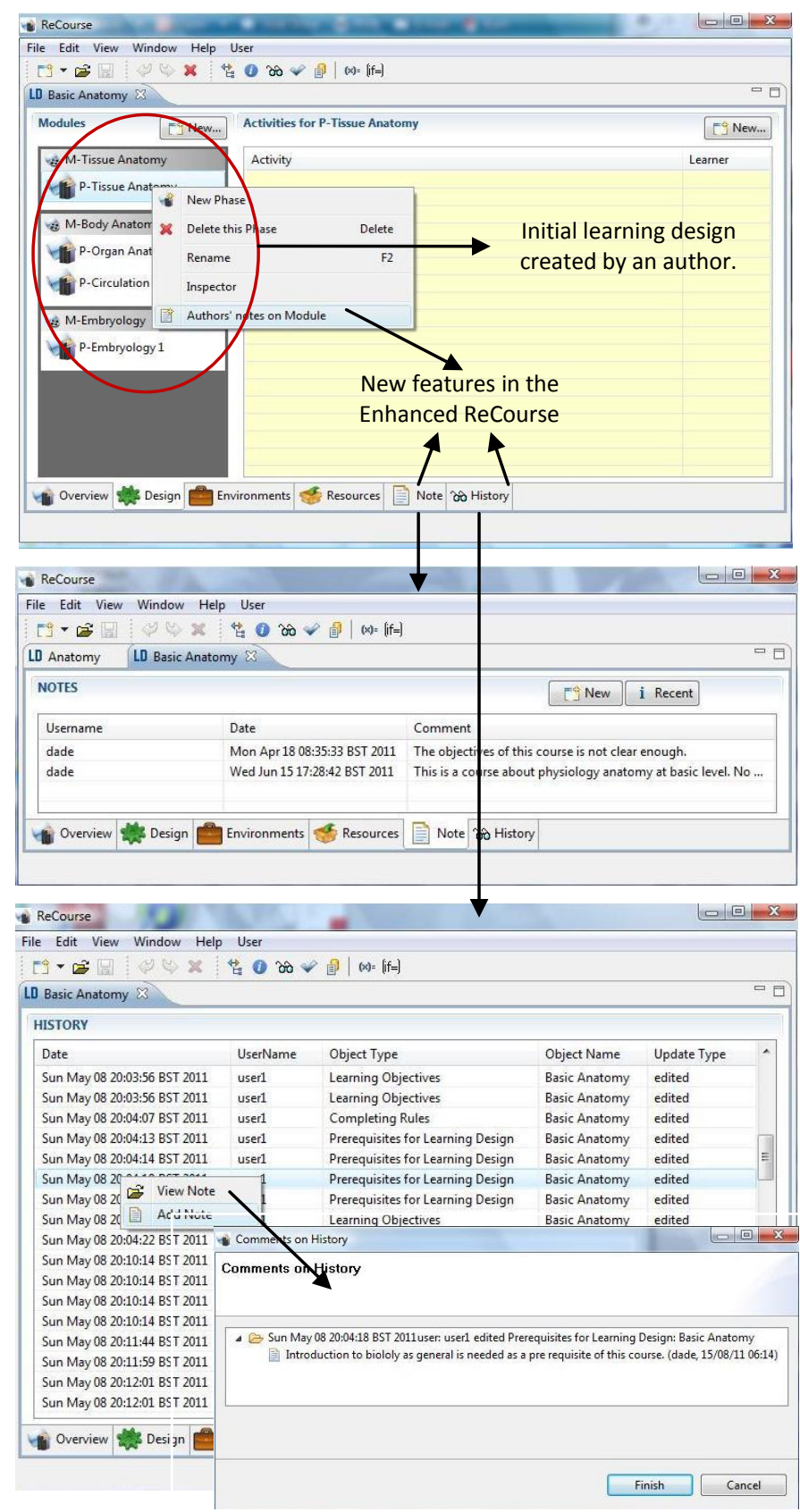

Figure 2. The Enhanced ReCourse for Collaborative Work

To be a collaborative work tool, ReCourse must be transformed into a web-based application with collaborative features that enable teachers or instructional designers to undertake asynchronously collaborative authoring. Note and History are both implemented in the learning design. Note is also implemented in authored objects and attached to each authored object. In addition, implicit coordination of planning is carried out by structuring the top levels of learning design, which consist of Modules and Phases created by an author. Then, all authors can update the structure. This kind of planning technique will prevent authors from wasting time with unnecessary discussions. 


\section{CONCLUSION AND FUTURE WORK}

A number of former research studies have established authoring tools with various authoring approaches. From a comparison analysis of the Collaborative ReCourse and existing authoring tools, three conclusions have been defined.

1. Adaptation Support. Since Collaborative (Enhanced) ReCourse uses IMS LD, it enables authoring of three kinds of adaptation: learning flow, content and problem-solving support.

2. Collaboration Support. In contrast to existing authoring tools, Collaborative ReCourse offers collaboration with some features, History and Note, to enhance authors' awareness. History implicitly describes how the interaction between authors and the system were made through updates, while Note explicitly shows the interactions among authors during the authoring process. All the authoring tools discussed above support the reuse of existing learning content.

3. Reuse. Collaborative ReCourse offers an advantage over other authoring tools by providing a learning content gallery that makes authors aware of existing resources. From these, authors can select the appropriate ones.

In future work, an evaluation through qualitative and quantitative inquiries will be carried out. This involves individuals who have experience in authoring for adaptive learning or collaborative work to prove that:

1. IMS LD is the most appropriate learning standard for adaptive learning resources in collaborative authoring environments.

2. In the context of authoring pedagogical materials, the incorporation of CSCW features in the forms of Note and History can enhance communication and group awareness among authors.

4. Applying a gallery of existing learning content available in open learning spaces, semantic wikis and wikis in general can enhance authors' awareness of the availability of related learning content.

\section{REFERENCES}

[1] Caplan, D., The development of online courses. The theory and practice of online learning, ed. T. Anderson and F. Elloumi: Athabasca, AB, Canada: Athabasca University. Retrieved January 7, 2011 from http://cde.athabascau.ca, 2004.

[2] Kearsley, G., Online education: learning and teaching in cyberspace: Belmont, CA: Wadsworth, 2000.

[3] Christensen, T.K. and R.T. Osguthorpe, "How do instructional-design practitioners make instructional-strategy decisions?" in Performance Improvement Quarterly, 2004, vol. 17(3): p. 45-65.

[4] Kirschner, P., C. Carr, J.v. Merrienboer, and P.B. Sloep, "How expert designers design", 2003.

[5] Kenny, R.F., Z. Zhang, R.A. Schwier, and K. Campbell, "A review of what instructional designers do: questions answered and questions not asked". in Canadian Journal of Learning and Technology, 2005, vol. 31(1).

[6] Kittur, A., B. Lee, and R.E. Kraut, "Coordination in collective intelligence: the role of team structure and task interdependence", in Proceedings of the 27th International Conference on Human Factors in Computing Systems, ACM: Boston, MA, USA, 2009.

[7] Kittur, A. and R.E. Kraut, "Harnessing the wisdom of crowds in wikipedia: quality through coordination", in Proceedings of the 2008
ACM Conference on Computer Supported Cooperative Work, ACM: San Diego, CA, USA, 2008.

[8] Ilaria Liccardi, H.C.D., Su White, "CAWS: Visualizing awareness to improve the effectiveness of co-authoring activities". in IEEE Distributed Systems Online, 2008, vol. 9(12): p. 1-6.

[9] Lowry, P.B., J.F. Nunamaker, Jr., Q.E. Booker, A. Curtis, and M.R. Lowry, "Creating hybrid distributed learning environments by implementing distributed collaborative writing in traditional educational settings". in IEEE Transactions on Professional Communication, 2004, vol. 47(3): p. 171 - 189.

[10] Liccardi, I., H.C. Davis, and S. White, "CAWS: a wiki system to improve workspace awareness to advance effectiveness of co-authoring activities", in CHI '07 extended abstracts on Human factors in computing systems, ACM: San Jose, CA, USA, 2007.

[11] Tudorache, T., N.F. Noy, S. Tu, and M.A. Musen, "Supporting collaborative ontology development in Protégé", in Proceedings of the 7th International Conference on The Semantic Web, Springer-Verlag: Karlsruhe, Germany, 2008.

[12] Dicheva, D., L. Aroyo, and A. Cristea, "Collaborative courseware authoring support ". in CATE '02: ACTA Pres, 2002.

[13] Ras, E., J. Rech, and S. Weber, "Collaborative authoring of learning elements for adaptive learning spaces", in 5th International Conference on Adaptive Hypermedia and Adaptive Web-Based Systems: Hannover, Germany, 2008.

[14] Posner, I.R. and R.M. Baecker, "How people write together [groupware]". in System Sciences, 1992. Proceedings of the TwentyFifth Hawaii International Conference on, 1992.

[15] Dourish, P. and V. Bellotti, "Awareness and coordination in shared workspaces", in Proceedings of the 1992 ACM conference on Computer-supported cooperative work, ACM: Toronto, Ontario, Canada, 1992.

[16] Weng, C. and J.H. Gennari, "Asynchronous collaborative writing through annotations", in Proceedings of the 2004 ACM conference on Computer supported cooperative work, ACM: Chicago, Illinois, USA, 2004.

[17] Viégas, F.B., M. Wattenberg, and K. Dave, "Studying cooperation and conflict between authors with history flow visualizations", in Proc. CHI 2004, ACM Press, 2004, p. 575-582.

[18] Ghali, F., A. Cristea, C. Stewart, and M. Hendrix, "Collaborative adaptation authoring and social annotation in MOT", Warwick Postgraduate Colloquium in Computer Science, 2008.

[19] Hixon, E., "Team-based online course development: a case study of collaboration models." in Online Journal of Distance Learning Administration, University of West Georgia, Distance Education Center., 2008, vol. 11(4).

[20] Kriplean, T., I. Beschastnikh, D. W. McDonald, and S. A. Golder, "Community, consensus, coercion, control: $\mathrm{cs}^{*} \mathrm{~W}$ or how policy mediates mass participation", in Proceedings of the 2007 international ACM conference on Supporting group work, ACM: Sanibel Island, Florida, USA, 2007.

[21] Nurjanah, D., H.C. Davis, and T. Tiropanis, "A Computer support collaborative authoring model for authoring adaptive educational hypermedia systems", in Web Science Conference: Raleigh, North Carolina, 2010

[22] Stewart, C., A. Cristea, and T.J. Brailsford, "Authoring once, delivering many: creating reusable adaptive courseware", in 4th IAESTED International Conference on Web Based Education (WBE '05): Grindewald, Switzerland, 2005.

[23] Kravcik, M., D. Burgos, M. Lebrun, and L. Oneto, "Integrated model of adaptive learning based on standards ", in GRAPPLE Deliverable 5.1 version 1.0, 2008.

[24] De Bra, P., A. Aerts, B. Berden, et al., "AHA! the adaptive hypermedia architecture", in the ACM Hypertext Conference: Nottingham, UK, 2006.

[25] Foss, J.G.K. and A.I. Cristea, "The next generation authoring adaptive hypermedia: using and evaluating the MOT3.0 and PEAL tools", in Proceedings of the 21st ACM conference on Hypertext and hypermedia, ACM: Toronto, Ontario, Canada.

[26] Hendrix, M., P.D. Bra, M. Pechenizkiy, D. Smits, and A. Cristea, "Defining adaptation in a generic multi layer model. CAM: the GRAPPLE conceptual adaptation model", in The Proceeding of ECTEL 2008, Springer, 2008, p. 132-143. 